\title{
Spatially-variant noise filtering in Magnetic Resonance Imaging: A Consensus-based approach
}

\author{
Luis González-Jaime*,a, Gonzalo Vegas-Sánchez-Ferreroc,d, Etienne E. Kerre ${ }^{\mathrm{a}}$, Santiago Aja-Fernández ${ }^{\mathrm{b}}$ \\ ${ }^{a}$ Applied Mathematics and Computer Science, Ghent University, Krijgslaan 281 - S9, 9000 Ghent, Belgium \\ ${ }^{b}$ Laboratorio de Procesado de Imagen, ETSI Telecomunicación, Universidad de Valladolid, 47011 Valladolid, Spain \\ ${ }^{c}$ Applied Chest Imaging Laboratory (ACIL), Brigham and Women's Hospital, Harvard Medical School, 1249 Boylston St., \\ Boston, MA 02115, USA \\ ${ }^{d}$ Biomedical Image Technologies Laboratory, ETSI Telecomunicación, Universidad Politécnica de Madrid, and CIBER-BBN, \\ Madrid, Spain
}

\begin{abstract}
In order to accelerate the acquisition process in multiple-coil Magnetic Resonance scanners, parallel techniques were developed. These techniques reduce the acquisition time via a subsampling of the $\mathbf{k}$-space and a reconstruction process. From a signal and noise perspective, the use of a acceleration techniques modify the structure of the noise within the image. In the most common algorithms, like SENSE, the final magnitude image after the reconstruction is known to follow a Rician distribution for each pixel, just like single coil systems. However, the noise is spatially non-stationary, i.e. the variance of noise becomes $\mathbf{x}$-dependent. This effect can also be found in magnitude images due to other processing inside the scanner. In this work we propose a method to adapt well-known noise filtering techniques initially designed to deal with stationary noise to the case of spatially variant Rician noise. The method copes with inaccurate estimates of variant noise patterns in the image, showing its robustness in realistic cases. The method employs a consensus strategy in conjunction with a set of aggregation functions and a penalty function. Multiple possible outputs are generated for each pixel assuming different unknown input parameters. The consensus approach merges them into a unique filtered image. As a filtering technique, we have selected the Linear Minimum Mean Square Error (LMMSE) estimator for Rician data, which has been used to test our methodology due to its simplicity and robustness. Results with synthetic and in vivo data confirm the good behavior of our approach.
\end{abstract}

Key words: MRI, Rician noise, SENSE, non-stationary distributed noise, consensus, noise filtering

\section{Introduction}

Magnetic Resonance Imaging (MRI) acquisitions suffers from different sources of degradations and artifacts that corrupt the original signal. One of the most dominant sources of degradation is noise. Thermal noise in MR scans is mainly originated by the subject or object to be imaged, followed by electronics noise during the acquisition of the signal in the receiver chain. Since noise is related to stochastic motion of free electrons, it is intrinsically imbricated with the acquisition process and therefore it is unavoidable. Some modern acquisition sequences are particularly affected by noise, like those ones in which the signal is attenuated, such as diffusion sequences with high b-values. It is also the case in those techniques that demand large amounts of data: in order to reduce the acquisition time, the number of excitations (NEX) is also reduced. As a consequence, the noise power is increased proportionally to the square root of the speedup.

\footnotetext{
* Corresponding author

Email addresses: luis.gonzalez@ugent.be (Luis González-Jaime), gvegsan@lpi.tel.uva.es (Gonzalo Vegas-Sánchez-Ferrero), etienne.kerre@ugent.be (Etienne E. Kerre), sanaja@tel.uva.es (Santiago Aja-Fernández)
} 
The degradation pattern introduced by noise affects the visual image quality and can negatively lead to an adequate interpretation and analysis of the data. Not only visual inspection is affected by the presence of noise, but also many common post-processing tasks (image registration, tissue segmentation, diffusion tensor estimation) and the obtaining of precise measures and quantitative imaging biomarkers.

The direct approach to minimize the influence of noise over the final image is the use of noise removal techniques, also kwon as denoising or, from a statisitical perspective, as signal estimation. Traditionally, noise filtering in medical imaging are based on well-defined prior statistical models of data. The Gaussian model is the usual assumption in many algorithms. The definition of more evolved noise models for MRI have allowed the natural extension of well-known image processing techniques to cope with features specific of MRI. Many examples can be found in the literature, such as the Conventional Approach (CA) (McGibney and Smith (1993)), Maximum Likelihood (ML) (Sijbers et al. (1998)), linear estimators (Aja-Fernández et al. (2008)), or adapted Non-Local Mean (NLM) schemes (Manjon et al. (2008); Tristán-Vega et al. (2012)).

In the simplest case, when single-coil acquisitions are considered, the complex spatial MR data is typically assumed to be a complex Gaussian process, where real and imaginary parts of the original signal are corrupted with uncorrelated Gaussian noise with zero mean and equal variance $\sigma^{2}$. Thus, the magnitude signal calculated as the envelope of the complex signal is known to be Rician distributed (Henkelman (1985); Gudbjartsson and Patz (1995)). This Rician model has been the standard in MRI modeling for many years, and it has been the base for a myriad of filtering techniques as well as noise estimation algorithms (Aja-Fernández et al. (2008); Manjon et al. (2008); Tristán-Vega et al. (2012); Luisier et al. (2012)).

With the advent of multiple-coil systems to reduce acquisition time, Parallel Magnetic Resonance Imaging (pMRI) algorithms are used, predominating among them Sensitivity Encoding (SENSE) (Pruessmann et al. (1999)) and GeneRalized Autocalibrating Partially Parallel Acquisitions (GRAPPA) (Griswold et al. (2002)). From a statistical point of view, the reconstruction process carried out by pMRI techniques is known to affect the spatial stationarity of the noise in the reconstructed data; i.e. the features of the noise become position dependent. Instead of assuming a single $\sigma^{2}$ value for each pixel within the image, the variance of noise varies with x, i.e. $\sigma^{2}(\mathbf{x})$ (Aja-Fernández et al. (2014); Aja-Fernández et al. (2015)).

If SENSE is considered, the reconstruction process yields to the magnitude value of a complex Gaussian, and therefore, the final magnitude signal can still be considered Rician distributed, but with a different $\sigma^{2}(\mathbf{x})$ for each $\mathbf{x}$ (Dietrich et al. (2008); Aja-Fernández et al. (2014); Aja-Fernández et al. (2015)). This way, many algorithms proposed for single coils systems can still be used if SENSE is considered, as long as the non-stationarity of the noise is taken into account. However, the estimation of the spatial pattern of $\sigma^{2}(\mathbf{x})$ is an issue that presents serious difficulties and some prior information is needed, such as the sensitivity maps in each coil. Unfortunately, this information is not always available. Recently, some estimation methods have adopted a non-parametric approach to estimate these non-stationary noise maps. These methods do not rely on a specific processing pipeline; the only requirement is that a statistical model has to be adopted for the acquisition noise: Gaussian, (Goossens et al. (2006); Pan et al. (2012); Aja-Fernández et al. (2015); Maggioni and Foi (2012)), Rician (Delakis et al. (2007); Liu et al. (2014); Aja-Fernández et al. (2015); Borrelli et al. (2014); Manjón et al. (2015)), or nc- $\chi$ (Tabelow et al. (2015); Pieciak et al. (2016)).

In this paper we propose a novel approach to noise filtering in MRI assuming non-stationary Rician noise in which the parameter $\sigma$ depends on the position, $\sigma(\mathbf{x})$. That is the case, for instance of SENSE acquisitions, but not only. It can also be found, for instance, in GRAPPA if the data from each coil is merged using a spatial matched filter instead of the sum of squares. The filtering method is based on the consensus of different realizations of a given signal estimator for different $\sigma^{2}$ values. The idea is to generate a wide variety of candidates that are merged in a global solution without the need of a $\sigma^{2}(\mathbf{x})$ estimation. Since the representative inputs are not known in advance, we use a set of aggregation functions to merge the realizations. Then, for each pixel, a penalty step will select the aggregated value that presents less dissimilarities with respect to the inputs, as proposed in Calvo et al. (2004); Calvo and Beliakov (2010). The final image is obtained with the information contained in the different candidates, showing a consistent spatially-variant behavior.

The work here presented is not a novel filtering method per se, but a methodology to adapt well-known statistical-based filters to a particular problem in which input parameters are unknown. It can be seen as an 
extension of the consensus framework for image processing proposed in two previous works: González-Jaime et al. (2013), where a general non-stationary Gaussian model where assumed; and in González-Jaime et al. (2014), where the uncertainty to deal with is the model of the noise that corrupts the image. The former approach deals with non-stationary noise in a similar way we do in this paper, while the latter considers stationary noise. The main advantage of the approach we propose in the current work, is that the existence of a well-defined prior noise model increases the amount of information available, which translates in a decrease of the uncertainty of the problem.

As a restoration algorithm, we consider the Linear Minimum Mean Square Error (LMMSE) estimator for Rician noise in Aja-Fernández et al. (2008), due to its simplicity and robustness, which is the natural extension of the Wiener filter to Rician noise. However, the method can be applied to other signal estimators.

The paper is organized as follows. Section 2 introduces the non-stationary Rician model in MRI as well as the LMMSE estimator and the consensus method. The aggregation and penalty functions are also explained. In Section 3 the proposed approach is presented. Then, in Section 4 different experiments are discussed for synthetic and real MR magnitude images using the new approach with LMMSE, to present our conclusions in Section 5.

\section{Background}

The method proposed in this paper is grounded in three different topics: (1) the non-stationary Rician model present in some MRI acquisitions; (2) The LMMSE estimator for Rician data and (3) the consensus methodology for decision taking when some information is missing. Next, we review the three of them.

\subsection{The non-stationary Rician noise model in MRI}

In MRI acquisitions, due to the reconstruction process and some post-processing done by the scanner, the noise in the final magnitude image can turn non-stationary, i.e. the variance of noise $\sigma^{2}$ becomes dependent on the position $\mathbf{x}: \sigma^{2}(\mathbf{x})$. This is the case when pMRI techniques are used.

Although the formulation of any specific pMRI method is beyond the scope of this work, as an illustration, let us assume that the reconstruction process combines the data of the different coils using a weighted sum to obtain the single complex image Aja-Fernández and Vegas-Sanchez-Ferrero (2016); Aja-Fernández et al. (2015):

$$
S^{\mathcal{R}}(\mathbf{x})=\sum_{l=1}^{L} \omega_{l}(\mathbf{x}) S_{l}^{\mathcal{S}}(\mathbf{x})
$$

where $\omega_{l}(\mathbf{x}), l=1, \cdots, L$ is a set of reconstruction weights that may depend on several parameters, such as the sensitivity of the coils; $S_{l}^{\mathcal{S}}(\mathbf{x})$ are the subsampled signals acquired in each coil and $S^{\mathcal{R}}(\mathbf{x})$ the reconstructed signal. This model, for instance, is the one we find in the case of pMRI data reconstructed with SENSE in its original formulation. The linear operations over the Gaussian data generate correlated Gaussian data, affecting the stationarity of the noise in the resulting image, which becomes corrupted with complex Additive Colored Gaussian Noise whose variance depends on the position (Aja-Fernández and Vegas-Sanchez-Ferrero (2016); Aja-Fernández et al. (2015)):

$$
S^{\mathcal{R}}(\mathbf{x})=A^{\mathcal{R}}(\mathbf{x})+N^{\mathcal{R}}\left(\mathbf{x} ; \sigma_{\mathcal{R}}^{2}(\mathbf{x})\right),
$$

where $N^{\mathcal{R}}\left(\mathbf{x} ; \sigma_{\mathcal{R}}^{2}(\mathbf{x})\right)=N_{r}^{\mathcal{R}}\left(\mathbf{x} ; \sigma_{\mathcal{R}}^{2}(\mathbf{x})\right)+j \cdot N_{i}^{\mathcal{R}}\left(\mathbf{x} ; \sigma_{\mathcal{R}}^{2}(\mathbf{x})\right)$ is no longer white, neither stationary. The final magnitude image is obtained by using the absolute value:

$$
M(\mathbf{x})=\left|S^{\mathcal{R}}(\mathbf{x})\right|
$$

and therefore it follows a non-stationary Rician distribution, with the parameter $\sigma_{\mathcal{R}}^{2}(\mathbf{x})$ being spatially variant. The specific value of $\sigma_{\mathcal{R}}^{2}(\mathbf{x})$ will depend on the reconstruction weights $\omega_{l}$ and on the covariance matrix $\boldsymbol{\Sigma}$. The final value of the variance of noise at each point will depend on the covariance matrix between coils of the original data (prior to reconstruction) and on the sensitivity map of each coil, but not on the 
data themselves. This model has been observed for SENSE by different authors through experimental and theoretical studies (see for instance the studies in Pruessmann et al. (1999); Thunberg and Zetterberg (2007); Robson et al. (2008); Aja-Fernández et al. (2014)).

The magnitude image can be modeled as follows:

$$
M(\mathbf{x})=\left|S^{\mathcal{R}}(\mathbf{x})\right|=\sqrt{\left(I_{0}(\mathbf{x})+N_{r}\left(\mathbf{x} ; 0, \sigma^{2}(\mathbf{x})\right)\right)^{2}+N_{i}\left(\mathbf{x} ; 0, \sigma^{2}(\mathbf{x})\right)^{2}},
$$

being $M(\mathbf{x})$ the noisy magnitude image, $I_{0}(\mathbf{x})$ a noise-free reconstructed signal and $N(\mathbf{x})=N_{r}(\mathbf{x})+j \cdot N_{i}(\mathbf{x})$ some complex Gaussian noise with zero mean and $\mathbf{x}$-dependent variance $\sigma^{2}(\mathbf{x})$.

\subsection{The LMMSE estimator for Rician noise}

The selected noise filtering technique is the LMMSE signal estimator for the stationary Rician distribution, as proposed in Aja-Fernández et al. (2008). It estimates the original signal $I_{0}(\mathbf{x})$ from the noise magnitude data, $M(\mathbf{x})$ that follows the model described in eq. (4), using the local information and the original variance of noise $\sigma^{2}$. The original estimator is defined over the square signal as follows

$$
\widehat{I_{0}^{2}}(\mathbf{x})=\left[K(\mathbf{x}) \cdot M^{2}(\mathbf{x})+(1-K(\mathbf{x})) \cdot\left\langle M^{2}(\mathbf{x})\right\rangle_{\mathbf{x}}\right]-2 \sigma^{2},
$$

with

$$
K(\mathbf{x})=1-\frac{4 \sigma^{2}\left(\left\langle M^{2}(\mathbf{x})\right\rangle_{\mathbf{x}}-\sigma^{2}\right)}{\left\langle M^{4}(\mathbf{x})\right\rangle_{\mathbf{x}}-\left\langle M^{2}(\mathbf{x})\right\rangle_{\mathbf{x}}^{2}}
$$

The operator $\left\langle M^{n}(\mathbf{x})\right\rangle_{\mathbf{x}}$ is the $\mathrm{n}$-th local sample moment of $M(\mathbf{x})$ in a neighborhood $\eta(\mathbf{x})$ around each pixel, defined as:

$$
\left\langle M^{n}(\mathbf{x})\right\rangle_{\mathbf{x}}=\frac{1}{|\eta(\mathbf{x})|} \sum_{\mathbf{p} \in \eta(\mathbf{x})} M^{n}(\mathbf{p}) .
$$

When non-stationary noise is considered, the parameter $\sigma^{2}$ becomes $\mathbf{x}$-dependent, and it must be replaced in eq. (5) and eq. (6) by $\sigma^{2}(\mathbf{x})$.

The function $K(\mathbf{x})$ in eq. (5) can be seen as a confidence measure of how data fits the considered model. In those pixels where $K(\mathbf{x}) \rightarrow 1$ (in the edges of the image, for instance, where the local variance is high), the data is far from the model, and therefore the final image $\widehat{I_{0}}(\mathbf{x}) \rightarrow M(\mathbf{x})-2 \sigma^{2}$. Since the model is not trusted, the output is just the data (with some bias removed). On the other hand, in those areas where $K(\mathbf{x}) \rightarrow 0$ (homogeneous areas, for instance), the model totally fits the data and the best possible output is given by an unbiased version of the averaged data, i.e., $\widehat{I}_{0}(\mathbf{x}) \rightarrow\left\langle M^{2}(\mathbf{x})\right\rangle_{\mathbf{x}}-2 \sigma^{2}$. This $K(\mathbf{x})$ function will be later used to control the consensus procedure.

\subsection{Decision based on Consensus}

A consensus strategy is used in a particular problem when the best solution among the possible ones is not known in advance. Thus, we choose the solution that produces less error among the provided solutions. With consensus techniques we can obtain a global solution that combines all the single inputs instead of using a single one as solution for the whole process (Calvo et al. (2004); Calvo and Beliakov (2010)). The main drawback of this decision-taking philosophy is that we have no prior information about whether all the candidates are representative or just some of them. This is our motivation to use a set of aggregation functions that previously merges the input candidates.

The whole consensus strategy consists in two phases: an aggregation phase and a selection phase. For the aggregation phase, we chose the family of parameterized averaging aggregation functions formed by the Ordered Weights Averaging (OWA) operators since they offer more flexibility when combining weighted information. An OWA operator of dimension $n$, defined by Yager in Yager (1988), is a mapping $F_{\mathbf{w}}$ : $[0,1]^{n} \rightarrow[0,1]$, where $\mathbf{w}=\left(w_{1}, \ldots, w_{n}\right) \in[0,1]^{n}$ with $\sum_{i=1}^{n} w_{i}=1$ and such that $F_{\mathbf{w}}\left(x_{1}, \ldots, x_{n}\right)=\sum_{j=1}^{n} w_{j} b_{j}$ 
with $\left\{b_{j}\right\}_{j=1}^{n}$ the sorted vector in decreasing order obtained from $\left\{x_{j}\right\}_{j=1}^{n}$. For instance, $b_{1}=\max _{j}\left(x_{j}\right)$ and $b_{n}=\min _{j}\left(x_{j}\right)$. Consequently, $y=F_{\mathbf{w}}\left(x_{1}, \ldots, x_{n}\right)$ is in $[0,1]$.

The possible operators to consider are unmanageable and we are not aware of the best candidate, so we can only assume a subset of operators based on our experience, $\left\{F_{\mathbf{w}_{j}}\right\}_{j=1}^{q}$. In other words, the choice of the different sets of $q$ aggregations to be used will depend on the specific problem under consideration.

From this set of $q$ aggregation functions and together with the inputs $\left(x_{1}, \ldots, x_{n}\right)$ we obtain a new set that corresponds with the possible outputs of our method, $\left\{y_{j}\right\}_{j=1}^{q}$. From the possible outputs, only one of the candidates can be used as a solution. Hence, in the selection phase, it is necessary to transform this set of outputs in only one that represents the largest number of inputs. For this purpose, a penalty function is used to select the aggregation value $y_{j}$ that minimizes the penalty with respect to the inputs and is given as a solution.

A penalty function measures the disagreement or dissimilarity between the $n$ candidates, $\left(x_{1}, \ldots, x_{n}\right)$, and the outputs of the $q$ aggregation functions, $\left\{y_{j}\right\}_{j=1}^{q}$. The penalty-based function (Calvo et al. (2004); Calvo and Beliakov (2010)) is defined as $P:[0,1]^{n+1} \rightarrow \mathbb{R}^{+}=[0, \infty)$ such that:

1. $P\left(x_{1}, \ldots, x_{n} ; y\right) \geq 0$ for all $x_{1}, \ldots, x_{n} \in[0,1], y \in[0,1]$;

2. $P\left(x_{1}, \ldots, x_{n} ; y\right)=0$ if $x_{i}=y$ for all $i=\{1, \ldots, n\}$;

3. $P\left(x_{1}, \ldots, x_{n} ; y\right)$ is quasi-convex in $y$ for any $\left(x_{1}, \ldots, x_{n}\right)$.

So, the consensus is achieved by calculating the minimum penalty among all the values obtained from the OWA operators:

$$
\hat{y}=\underset{y \in\left\{y_{j}\right\}_{j=1}^{q}}{\operatorname{argmin}} P\left(x_{1}, \ldots, x_{n} ; y\right) .
$$

So, we can consider any function $P(\mathbf{x}, y)$ that meets the three exposed conditions. Among the possible functions that could be suitable for this problem (Calvo and Beliakov (2010)) we have used $P(\mathbf{x}, y)=$ $\sum_{j=1}^{n}\left|x_{j}-y\right|$. Other functions could also be used in this problem, as previously studied in González-Jaime et al. (2014). Alternative functions can be found in Liu et al. (2013) and Beliakov and James (2014).

Accordingly, a consensus strategy is based on testing several functions until we find the one providing the least dissimilar result with respect to the values of the inputs. Moreover, as we can expect the result provided depends on the set of $q$ aggregation functions and the penalty function $P(\mathbf{x}, y)$ that we use in each case.

\section{A consensus-based LMMSE filter for MRI data}

For our work we will consider those MRI signals corrupted with non-stationary Rician noise, as those generated after a SENSE acceleration and reconstruction. Our aim is to estimate the original signal (without noise) from the original data without any knowledge of the value of parameter $\sigma(\mathbf{x})$. To that end, we will assume the non-stationary noise model described in eq. (4). Although many strategies can be adopted, we have selected the LMMSE estimator in eq. (5) as the filtering technique, because of its simplicity and for having a formulation that can be directly adapted to the consensus techniques. As an initial assumption, we consider that the value of the noise $\sigma^{2}(\mathbf{x})$ cannot be accurately estimated from the data. Thus, we cannot initially calculate a value for $K(\mathbf{x})$ in eq. (6), since it depends on this parameter.

The solution proposed to overcome this issue is the use of a consensus strategy to a pixel level: from a set of different input values of $K_{i}(\mathbf{x}), i=1, \ldots, n$ we try to reach a consensus for an unique $K(\mathbf{x})$ value:

$$
\left[\begin{array}{c}
K_{1}(\mathbf{x}) \\
K_{2}(\mathbf{x}) \\
\vdots \\
K_{n}(\mathbf{x})
\end{array}\right] \stackrel{\text { Consensus }}{\longrightarrow} K(\mathbf{x})
$$




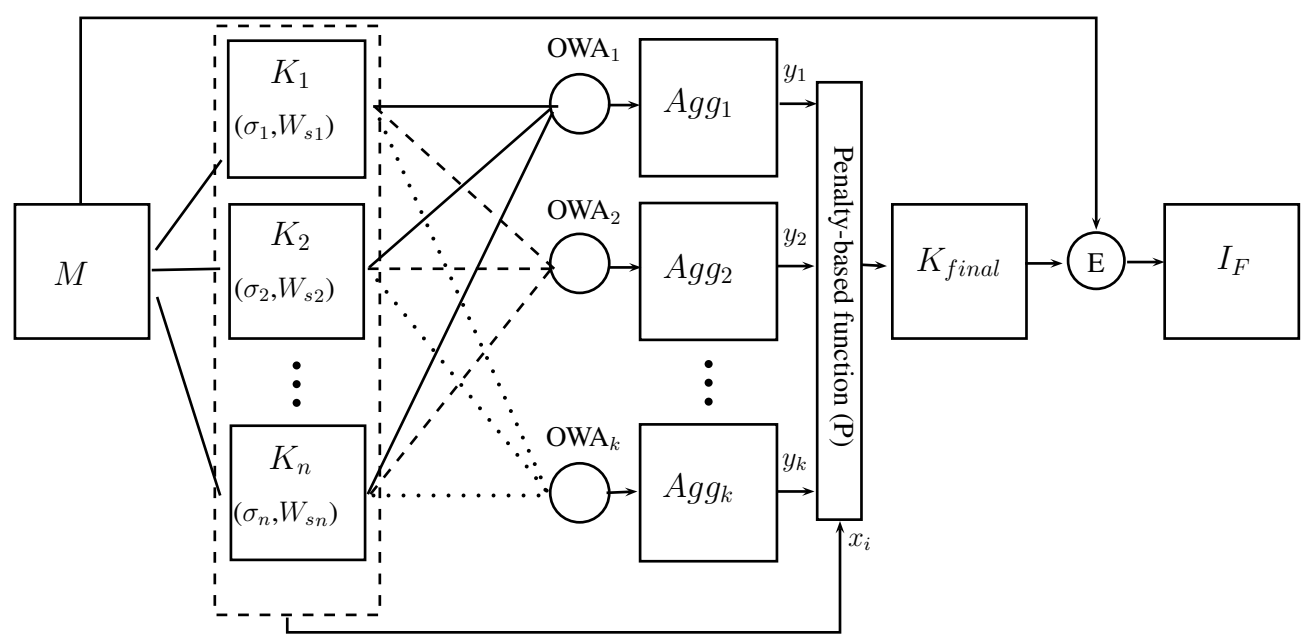

Figure 1: Proposed scheme for filtering of nonstationary noise using a LMMSE estimator for Rician noise. A consensus approach for multiple inputs as a function of $K(\mathbf{x})$ is considered.
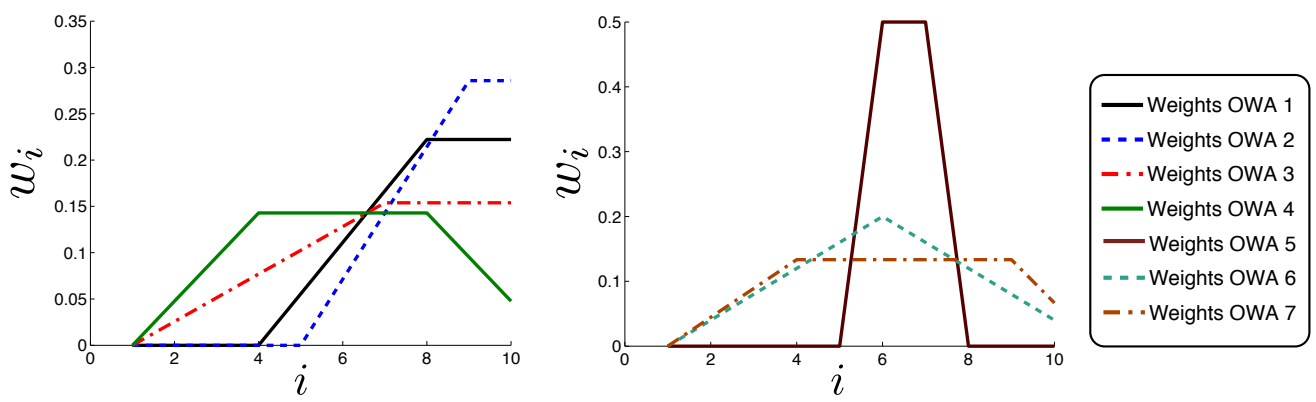

Figure 2: Weighting quantification for the 7 OWA operators used in the paper, considering 10 elements.

The different $K_{i}(\mathbf{x})$ are calculated using different configurations of the input parameter set, namely a $\sigma^{2}$ value and the size of the neighborhood where the local moments are calculated, $W_{s i}=\left|\eta_{i}(\mathbf{x})\right|$, see eq. (7). The function $K(\mathbf{x})$ here is used as a pixel confidence: it gives a measure of how the data fits the model. Since an initial estimation of $\sigma^{2}(\mathbf{x})$ is not available, different candidates $K_{i}(\mathbf{x})$ calculated with different $\sigma_{i}^{2}$ values will contribute to the final decision.

The complete scheme of the proposed method is depicted in Fig. 1. The whole consensus-based algorithm is as follows:

1. A set of confidence matrices $\left\{K_{i}\right\}_{i=1}^{n}$ is calculated using eq. (6) with different values for the noise variance $\left(\sigma_{i}^{2}\right)$ and the neighborhood size $\left(W_{s i}\right)$. A reference set $\left\{\sigma_{i}^{2}\right\}_{i=1}^{n}$ can be built from an initial reference variance. For instance, a reference variance can be estimated using any noise estimator already existing in the literature (Aja-Fernández et al. $(2008,2009)$ ). This estimation is done assuming a single $\sigma^{2}$ value for the whole image, which will not be accurate for all pixels, but it gives a global reference value. A set of multiple $\left\{\sigma_{i}^{2}\right\}_{i=1}^{n}$ can be obtained by sampling an inter-percentile interval around the estimated value. Other strategies can be also adopted when some information on the underlying variance is known.

2. A set of aggregation functions merges all the information from $\left\{K_{i}\right\}_{i=1}^{n}$. Then a set of aggregated confidence matrices $\left\{\mathrm{Agg}_{j}\right\}_{j=1}^{k}$ is generated by applying OWA operators with different weighting vectors. A set of seven representatives OWA operators was used, whose weighting vectors are depicted in Fig. 2. Note that the weights distributions follow trapezoidal shapes with different tilt grades. They 


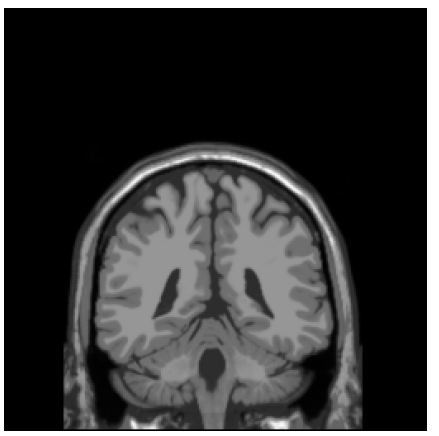

(a) Coronal $(512 \times 512)$

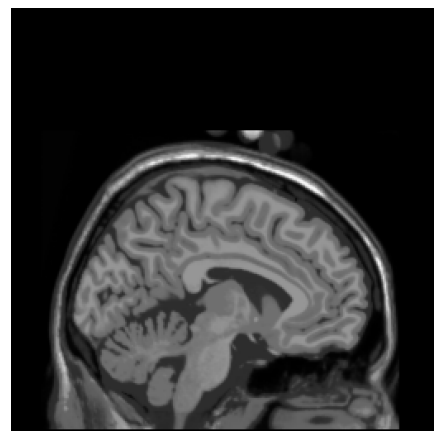

(b) Sagital $(512 \times 512)$

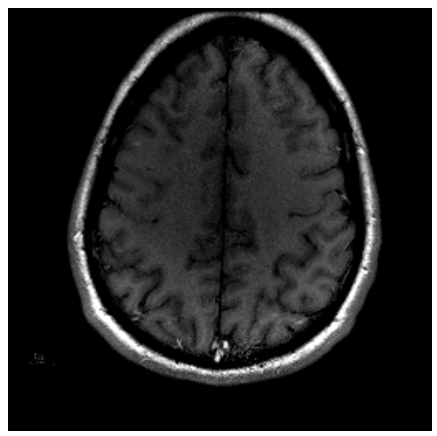

(c) In vivo $(256 \times 256)$

Figure 3: MRI slices used in the experiments. Images (a) and (b) comes from the BrainWeb dataset; (c) is a real in vivo acquisition from a multi-coil GE Signa 1.5T EXCITE.

give higher weights to the lower values of the sorted input. This way, the output of the OWA operator provides a higher confidence value when the majority of candidates agree. There are also null weights that correspond to the input omission.

3. In order to build $K_{\text {final }}(\mathbf{x})$, we select the $\operatorname{Agg}_{j}$ that best suits and less disagrees with respect to the initial $\left\{K_{i}\right\}_{i=1}^{n}$. In order to help in this issue the $\mathrm{Agg}_{j}$ is calculated by minimizing the penalty-based function

$$
K_{\text {final }}(\mathbf{x})=\operatorname{argmin}_{\mathrm{Agg}} \sum_{i=1}^{n}\left|K_{i}(\mathbf{x})-\operatorname{Agg}_{j}(\mathbf{x})\right| .
$$

4. Finally, the estimation of the original signal is calculated using the LMMSE estimator from eq. (5), using the confidence estimation $K_{\text {final }}(\mathbf{x})$ and a spatial variance estimation $\widehat{\sigma^{2}}(\mathbf{x})$ calculated from eq. (6) as:

$$
\widehat{\sigma^{2}}(\mathbf{x})=\frac{\left\langle M^{2}(\mathbf{x})\right\rangle_{\mathbf{x}}-\sqrt{\left\langle M^{2}(\mathbf{x})\right\rangle_{\mathbf{x}}^{2}-\left(1-K_{\text {final }}(\mathbf{x})\right) \cdot\left(\left\langle M^{4}(\mathbf{x})\right\rangle_{\mathbf{x}}-\left\langle M^{2}(\mathbf{x})\right\rangle_{\mathbf{x}}^{2}\right)}}{2} .
$$

\section{Experiments}

\subsection{Materials and methods}

We tested the proposed method with two different data sets as it is shown in Fig. 3: (1) Synthetic noise-free MR slices from the BrainWeb data set (Cocosco et al. (1997)); (2) one in vivo T1 MR magnitude image acquired in a GE Signa 1.5T EXCITE, FSE pulse sequence, 8 coils, TR=500ms, TE $=13.8 \mathrm{~ms}$, image size $256 \times 256$ and FOV: $20 \mathrm{~cm} \times 20 \mathrm{~cm}$.

First, the synthetic images are corrupted with non-stationary noise following the model in eq. (4). Three different spatial patterns, $\mathcal{G}(\mathbf{x})$ are considered to model the spatial variation of $\sigma(\mathbf{x})$, shown in Fig. 4 . The noise variance is calculated from these patterns for different signal-to-noise ratio (SNR) simply by a linear scaling:

$$
\sigma(\mathbf{x})=\sigma_{0}+\mathcal{G}(\mathbf{x}) \cdot \sigma_{1}
$$

where $\sigma_{0}$ and $\sigma_{1}$ are constants. The different patterns used are:

1. An unrealistic highly variant synthetic noise pattern, Fig. 4-(a). Although it is very unlikely that a pattern like this occurs in real acquisition, this 4-section scheme will give a very good insight of the behavior of the filtering schemes. 


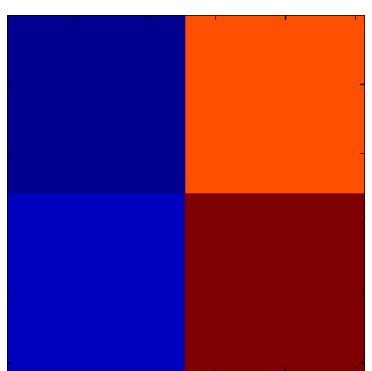

(a) Extreme

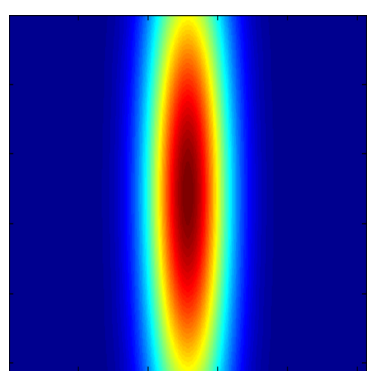

(b) Slim

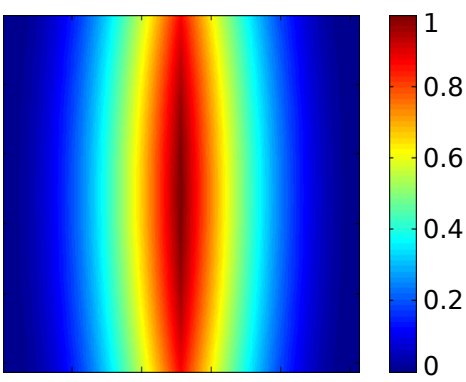

(c) SENSE

Figure 4: Non-stationary noise patterns used with the synthetic MR images. $\mathcal{G}(\mathbf{x})$ range is $[0,1]$. It was scaled to obtain images with several SNRs.

2. A synthetic Gaussian-shaped noise pattern, Fig. 4-(b). This pattern follows the shape of some real patterns found in SENSE acquisitions (Aja-Fernández et al. (2014); Aja-Fernández and Vegas-SanchezFerrero (2016)).

3. A noise shape generated with a SENSE simulator: Fig. 4-(c). This is the reconstruction from a sensitivity map belonging to 8-coils scheme.

For the experiments, a neighborhood size $W_{s}=[7 \times 7]$, and a range of 10 different central values for $\sigma^{2}$ are considered. Our approach was compared with the following state-of-the-art Rician-based filtering schemes:

- The original LMMSE estimator (Original LMMSE) as proposed in Aja-Fernández et al. (2008), assuming a single $\sigma^{2}$ value for the whole image. A $7 \times 7$ square window is used for the sample moments estimation.

- The Non-local-mean (NLM) algorithm without the Rician bias, as proposed in Manjon et al. (2008) (Rice NLM). The essence of the NLM algorithm consists on a weighted average that considers the distance and intensity between the target pixel and all observed pixels. The original idea was proposed by Buades et al. (2005) for Gaussian noise. The required parameters for this approach are the radio search window $\left(R_{\text {search }}=11\right)$; the radio similarity window $\left(R_{\text {sim }}=3\right)$; the degree of filtering $(f=1.2 \cdot \widehat{\sigma})$ and an estimation of the variance $\left(\widehat{\sigma^{2}}\right)$.

- The Chi-square unbiased risk estimator (CURE), as proposed in Luisier et al. (2012). It considers the squared-magnitude magnetic resonance image data to derive an unbiased expression for the expected mean-squared error to remove noise, which are well modeled as independent non-central chi-square random variables on two degrees of freedom. The task is done in the wavelet-domain for its compromise between the execution speed and performance. It uses the unnormalized Haar wavelet transform (Haar CURE), where each wavelet subband is treated independently. The other required parameter is the variance estimation, $\widehat{\sigma^{2}}$.

For the sake of comparison, the following methods will also be used:

- The original LMMSE with the actual value of the noise map $\sigma^{2}(\mathbf{x})$, denoted by Ideal LMMSE, This method represents the upper bound of the proposal: it is the best possible solution the LMMSE can achieve.

- The LMMSE described in eq. (5) needs an estimate of $\sigma^{2}(\mathbf{x})$ to remove the bias of the estimated signal and a value of $K_{\text {final }}(\mathbf{x})$ for filtering. Thus, the accuracy of the final estimated signal will depend on these two parameters. In order to isolate the behavior of the filtering and the bias reduction steps, we 

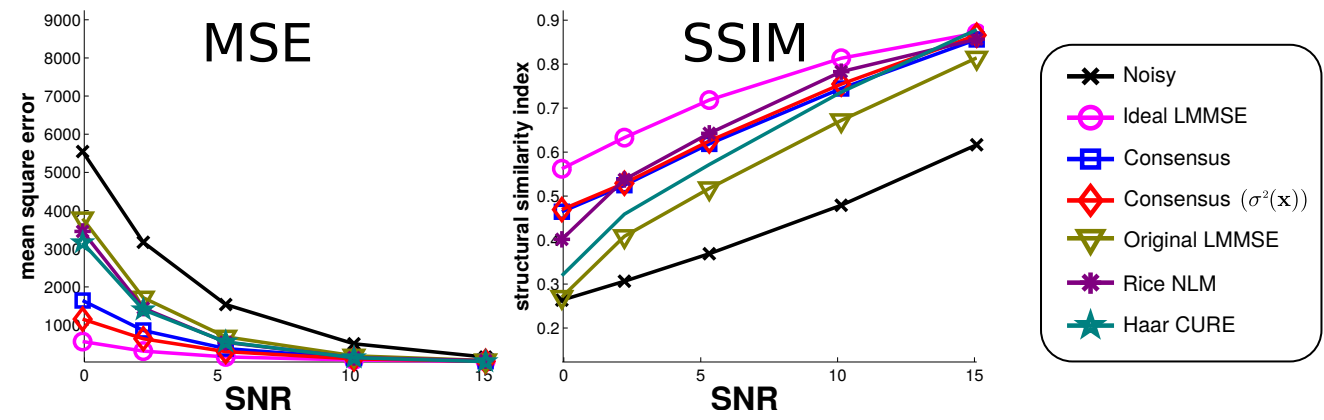

Figure 5: Results from the first experiment: the synthetic MR magnitude image from Fig. 3-(a) is corrupted with Rician noise with different SNRs generated with the noise map in Fig. 4-(a). The average of 100 realizations is considered.

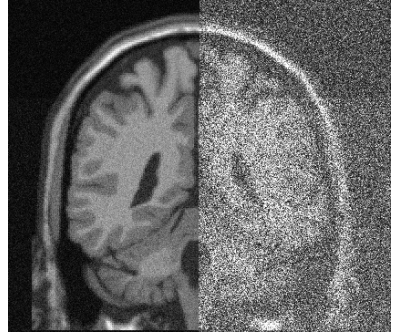

(a) Noisy

$\mathrm{MSE}=3174, \mathrm{SSIM}=0.31$

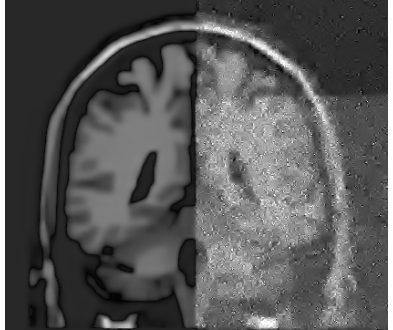

(b) CURE

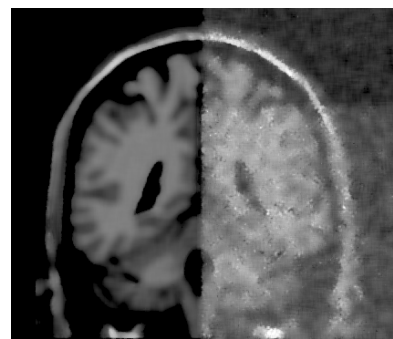

(c) Rice NLM

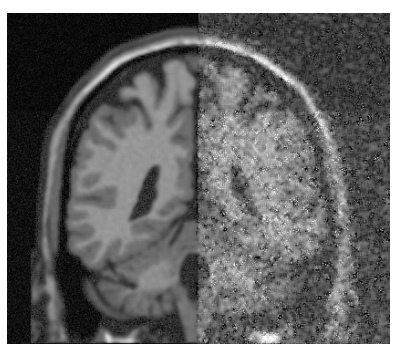

(d) Consensus

$$
\mathrm{MSE}=1410, \mathrm{SSIM}=0.46 \quad \mathrm{MSE}=1440, \mathrm{SSIM}=0.54 \quad \mathrm{MSE}=874, \mathrm{SSIM}=0.52
$$

Figure 6: Visual results of the filtered image obtained from the synthetic MR magnitude image from Fig. 3-(a) with the extreme noise map in Fig. 4-(a), with a $\mathrm{SNR}=2.21$.

will show the results of the complete approach together with those in which the $K_{\text {final }}(\mathbf{x})$ is used, but the bias is removed with the original $\widehat{\sigma^{2}}(\mathbf{x})$. The former will be label as Consensus, and the latter as Consensus $\left.\widehat{\sigma^{2}}(\mathbf{x})\right)$.

The restoration performance was quantified by using the Mean Square Error (MSE) and the Structural Similarity Index (SSIM) by Wang et al. (2004). The former measure is used to quantify the error reduction due to the filtering. It is not bounded and a higher MSE means worse quality. On the other hand, the SSIM index gives a measure of the structural similarity between the ground truth and the estimated image. It is bounded in $[0,1]$; the closer to one, the better the image. Both measures are only applied on areas of interest in the image, this means that the background is excluded. Each experiment is repeated 100 times to present a significant statistical analysis.

\subsection{Experiments with synthetic data}

The first experiment evaluates the behavior in an extreme case with an unreal noise shape. To that end the coronal synthetic slice in Fig. 3-(a) is corrupted with a non-stationary noise following the unrealistic map in Fig. 4-(a). Results can be found in Fig. 5. Note that most of the methods show a poor behavior for low SNR, being the Ideal LMMSE the one with the smaller MSE and the highest SSIM. Our proposal shows also a good behavior that converges to the ideal one for high SNR. However, note that the method shows some error due to the the removal of the bias: the Consensus that uses the original $\sigma^{2}(\mathbf{x})$ is slightly better in terms of MSE, but very similar in terms of SSIM. This means that there is some numerical error, but the underlying structure is preserved. In Fig. 6 we show a visual example for $\mathrm{SNR}=2.21$. Most of the methods works poorly on the areas with the higher noise values, but the proposal keeps the structures without any over-blur of the edges. 

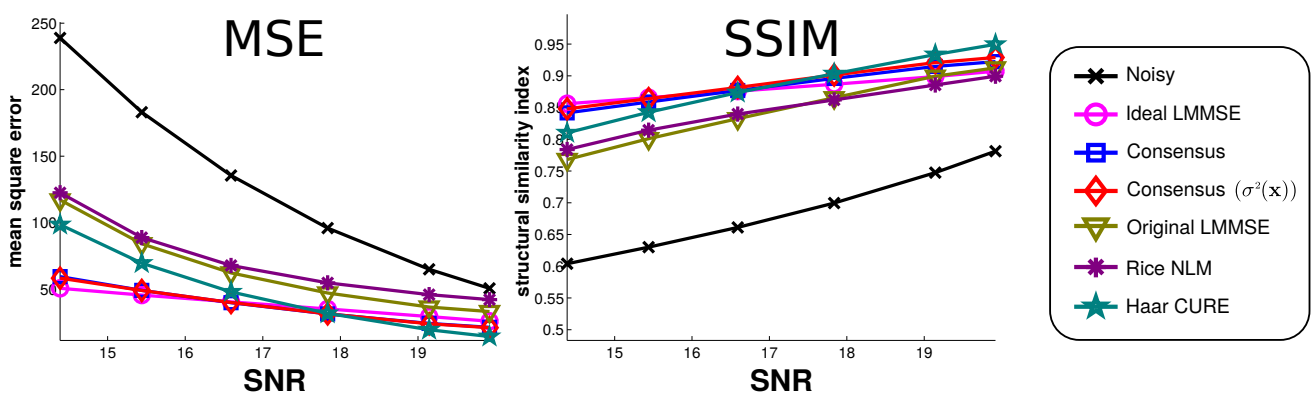

Figure 7: Results from the second experiment: the synthetic sagittal MR magnitude slice from Fig. 3-(b) is corrupted with Rician noise with different SNRs generated with the em slim noise map in Fig. 4-(b). The average of 100 realizations is considered.

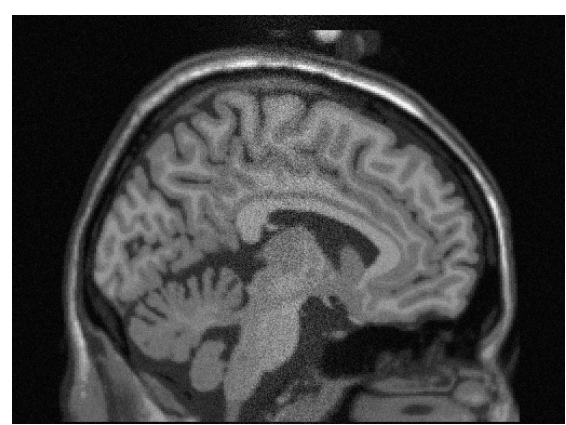

(a) Noisy $(\mathrm{MSE}=135.74, \mathrm{SSIM}=0.66)$

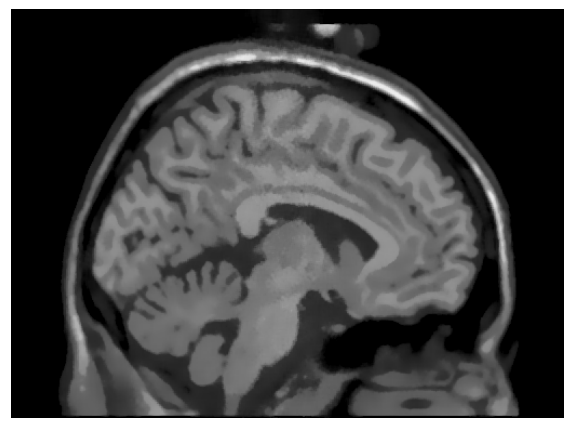

(c) Rice NLM (MSE $=67.97, \mathrm{SSIM}=0.84)$

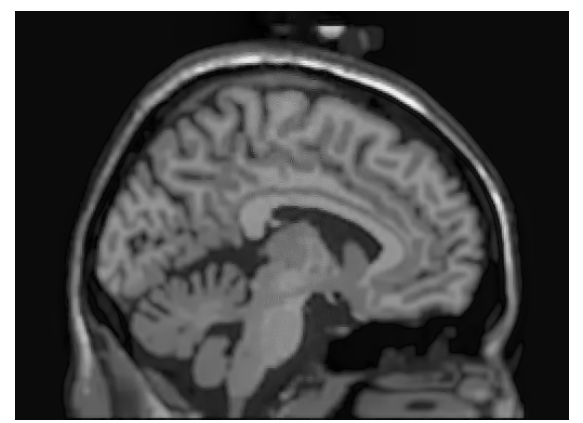

(b) $\mathrm{CURE}(\mathrm{MSE}=48.86, \mathrm{SSIM}=0.87)$

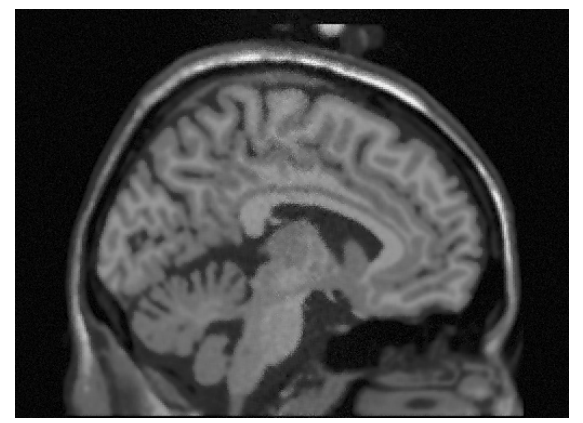

(d) Consensus $(\mathrm{MSE}=40.02, \mathrm{SSIM}=0.88)$

Figure 8: Visual results of the filtered image obtained from the synthetic MR magnitude image from Fig. 3-(b) with the slim noise map in Fig. 4-(b), with a SNR = 16.59.

In the second experiment, the sagittal synthetic slice from Fig. 3-(b) is corrupted with Rician noise. This time, we adopt a more realistic pattern for $\sigma(\mathbf{x})$, the slim noise map in Fig. 4-(b). Due to the shape of this map, in which there is a great decay of the values of $\sigma(\mathbf{x})$ far from the center, the resulting SNR values are higher. Results for different SNRs are depicted in Fig. 7, and a visual example for SNR $=16.59$ can be found in Fig. 8. Due to the higher levels of SNR, the behavior of all the filters improve, when compared to those of the first experiment. This time, the Consensus approach quickly converges to the ideal value. When the SNS increases, CURE becomes better than to our approach. However, note that the consensus approach is limited by the selected filter, the LMMSE in this case. Nevertheless, in all the cases, our approach improves the original LMMSE with a single $\sigma$ value.

For the third experiment, a SENSE reconstruction is simulated from the synthetic slice in Fig. 3-(a). 

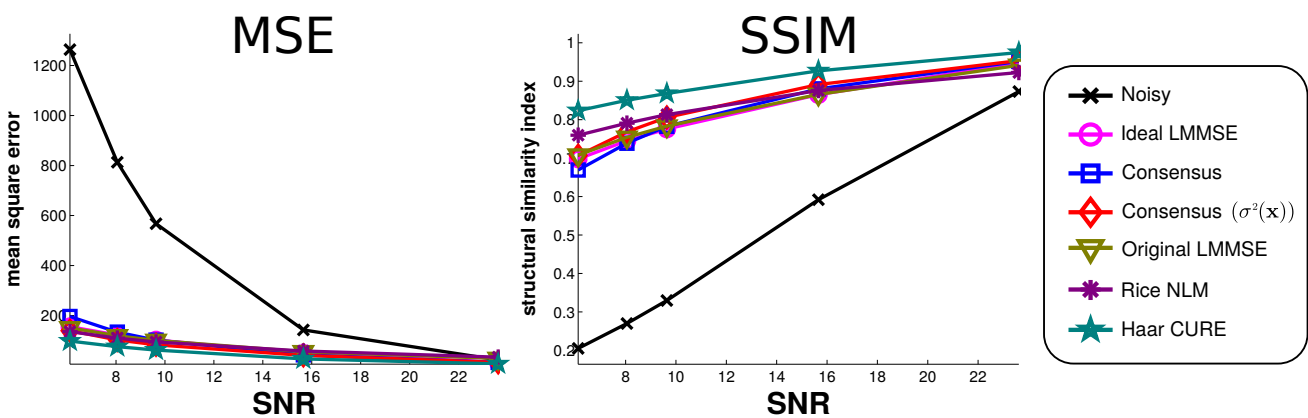

Figure 9: Results from the third experiment: the synthetic sagittal MR magnitude slice from Fig. 3-(b) is used to simulate a SENSE reconstruction with different SNRs. The average of 100 realizations is considered.

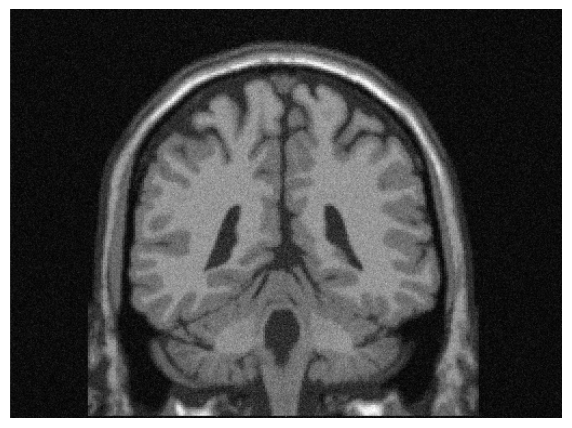

(a) Noisy $(\mathrm{MSE}=141, \operatorname{SSIM}=0.59)$

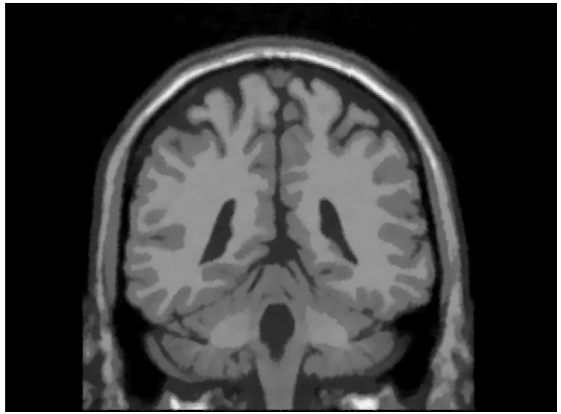

(c) Rice NLM (MSE $=58$, SSIM $=0.88)$

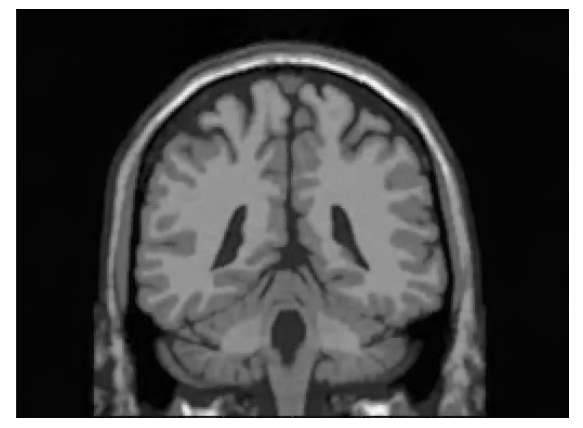

(b) CURE (MSE $=27$, SSIM $=0.93)$

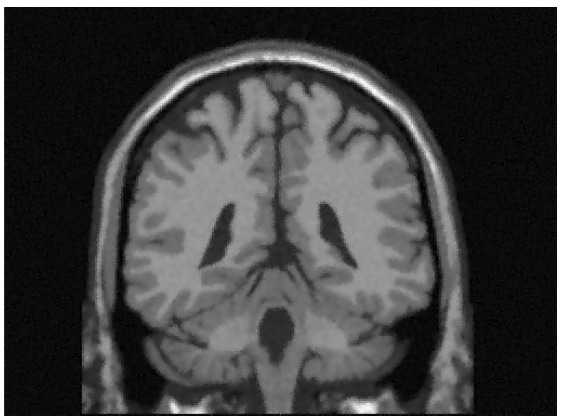

(d) Consensus $(\mathrm{MSE}=42, \operatorname{SSIM}=0.88)$

Figure 10: Visual results of the filtered image obtained from a SENSE image, generated from the synthetic MR magnitude image from Fig. 3-(a), with a $\mathrm{SNR}=15.66$. An 8-coil system is considered.

An 8-coil system is simulated using artificial sensitivities for each coil, so that the image in the $l$-th coil can be seen as Aja-Fernández et al. (2014)

$$
I_{l}(\mathbf{x})=C_{l}(\mathbf{x}) \times I_{0}(\mathbf{x}), \quad l=1, \cdots, 8,
$$

with $C_{l}(\mathbf{x})$ the sensitivity map in the $l$-th coil. Each coil is corrupted with Gaussian noise with a single $\sigma$ value, and a correlation between coils is assumed $(\rho=0.25)$. The $\mathbf{k}$-space is then subsampled by a factor $2 \times$ and reconstructed using Cartesian SENSE. Different values of $\sigma$ are considered. The resulting magnitude signal has a noise map similar to the one in Fig. 4-(c). Results for different SNRs are depicted in Fig. 9 while a visual example can be found in Fig. 10. This time, most of the methods show a similar behavior, with small differences between them in terms of MSE. Those differences are clearer for the SSIM index. We can clearly appreciate that CURE outperforms the rest of the approaches, followed by the NLM. Once more, 

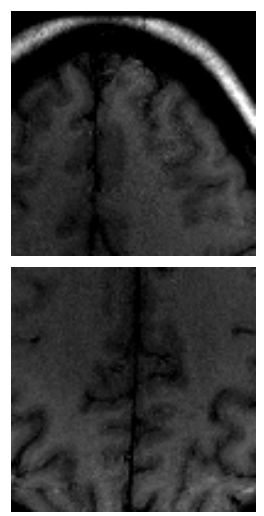

(a) Original
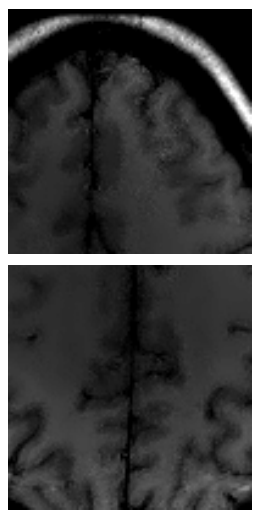

(b) Consensus
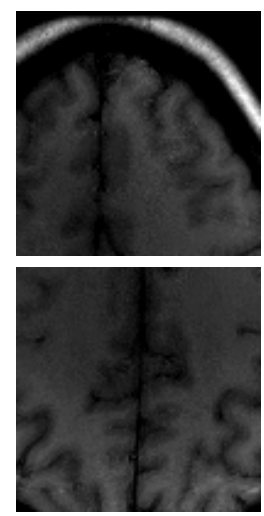

(c) LMMSE
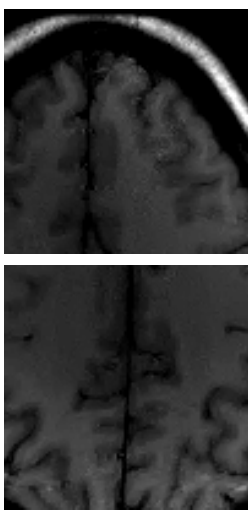

(d) Haar CURE
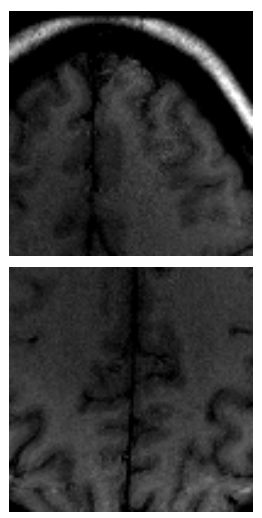

(e) Rice NLM

Figure 11: Visual results for the in vivo data set in Fig. 3-(c): details from the filtered images.

note that the performance of the Consensus approach is limited by the selected filter. Note also that the case without noise estimation totally converges into the ideal one. On the other side, it is important to note that, as the SNR increases, the performance differences decrease between the algorithms.

\begin{tabular}{l|r|r|r} 
Experiment & 1 (Fig. 5) & 2 (Fig. 7) & 3 (Fig. 9) \\
\hline \hline Original LMMSE & $0.061 \mathrm{~ms}$ & $0.072 \mathrm{~ms}$ & $0.060 \mathrm{~ms}$ \\
Consensus & $1.302 \mathrm{~ms}$ & $1.405 \mathrm{~ms}$ & $1.306 \mathrm{~ms}$ \\
Haar CURE & $5.828 \mathrm{~ms}$ & $6.710 \mathrm{~ms}$ & $5.727 \mathrm{~ms}$ \\
Rice NLM & $87.106 \mathrm{~ms}$ & $96.602 \mathrm{~ms}$ & $80.173 \mathrm{~ms}$
\end{tabular}

Table 1: Average running times for 100 executions of the different algorithms and different experiments carried out.

Finally, we want to point out that this consensus-based approach not only shows a great behavior dealing with non-stationary noise, but it also exhibits good running times. In Table 1 we present the average time of the 100 executions considered for each algorithm ${ }^{1}$. Not that the original LMMSE is a very fast method, closely followed by our approach. On the other hand, the Rice NLM obtains high running times with respect to the rest of the filters. This issue must be taken into account if the filters are going to be used in large data sets.

\subsection{Experiments with real data}

Finally, in order to test the proposed method with real data, we use the multicoil in vivo acquisition in Fig. 3-(c). For simplicity, the fully sampled $\mathbf{k}$-space has been acquired, and the sensitivity map has been estimated for each of its 8 coils. The data in each coil was subsampled to simulate a $2 \times$ acceleration, and the final magnitude image has been reconstructed using an offline SENSE algorithm. Since the initial $\sigma^{2}(\mathbf{x})$ is not available for this image, a prior estimation is done assuming stationary noise (Aja-Fernández et al. (2008)) as

$$
\widehat{\sigma^{2}}=\operatorname{mode}\left\{\left\langle M(\mathbf{x})^{2}\right\rangle_{\mathbf{x}}\right\} .
$$

The $\left\{\sigma_{m}^{2}\right\}_{m=1}^{10}$ elements are selected from the range $1 \%$ to $103 \%$ of the estimated $\widehat{\sigma}$, that is, the interval $\left[0.0001 \cdot \widehat{\sigma^{2}},, 1.0609 \cdot \widehat{\sigma^{2}}\right]$. Since there is no golden standard available for comparison, we only show visual results in Fig. 11 for the different filters considered. The consensus LMMSE and the Haar CURE obtain

\footnotetext{
${ }^{1}$ The experiments were done in MATLAB and run on a Intel Core i5 $2.4 \mathrm{GHz}$ with 8GB of RAM.
} 
the best results among the restored images, although consensus LMMSE slightly obtains better results in homogeneous areas. On the other hand, the restored Rice NLM image still keeps some noisy pattern, while the original LMMSE over-filter the data, producing some blur (this effect is emphasized close to the borders).

\section{Conclusions}

A new methodology is presented as a solution to noise filtering when the input image shows a spatially variant noise pattern, and some of the input variables cannot be properly estimated. Spatial non-stationary noise is a kind of noise whose features (the variance in this case) depends on the position within the image. The clearer example of this kind of noise in MR data can be found in pMRI acquisitions, but not only. The proposed method is applied together with some existing filtering method. In this paper, the LMMSE signal estimator for stationary Rician noise is considered. This filter on its own will fail when applied over a spatially variant $\sigma^{2}(\mathbf{x})$, since it is intended for a single $\sigma^{2}$. However, the combination of the LMMSE with the proposed consensus approach is able to take into account the non-stationarity of the data. The algorithm also assumes our incapability to proper estimate the input data, in this case the map of noise and the optimal size of the window in which the local moments are calculated.

Results of the experiments done using synthetic and real data show how the proposed method highly improves the behavior of the stationary LMMSE, and its performance is very similar to the optimal case assuming a non-stationary LMMSE with $\sigma^{2}(\mathbf{x})$ perfectly known. In many cases, the new approach even outperforms Rician filters that in the past have shown even a better performance than the LMMSE itself. The method is particularly useful in those cases when the variability of $\sigma^{2}(\mathbf{x})$ is high and extreme. That will depend on the position and calibration of the acquisition coils.

As we have previously stated, this philosophy of work can be easily extended to other filtering techniques in MRI. This extension will allow other algorithms to better cope with non-stationary noise, but not only. They can also be adapted to automatically select the better set of input parameters, or to cope with deviation from the statistical model, to perform a different filtering around important structures and edges or even to combine the results of different kind of filters into a single output.

The main drawback of the method is that the number of operations increases, since the method carries out a filtering procedure for each input set. The more the input possibilities, the greater the number of times the filtering is repeated. The good news here is that each of the iterations is totally independent of the others, and therefore the method can easily be highly parallelized.

\section{Acknowledgements}

This work was supported by European Commission under Contract no. 238819 (MIBISOC Marie Curie ITN) and Ministerio de Ciencia e Innovación for grant TEC2013-44194. The authors also acknowledge Centro de Técnicas Instrumentales, Universidad de Valladolid and Doctor W. Scott Hoge from Brigham and Womens Hospital, Boston for MRI acquisitions. Gonzalo Vegas-Sánchez-Ferrero acknowledges Consejería de Educación, Juventud y Deporte of Comunidad de Madrid and the People Programme (Marie Curie Actions) of the European Union's Seventh Framework Programme (FP7/2007-2013) for REA grant agreement no. 291820 .

\section{References}

Aja-Fernández, S., Alberola-López, C., Westin, C.F., 2008. Noise and signal estimation in magnitude MRI and Rician distributed images: A LMMSE approach. Image Processing, IEEE Transactions on 17, 13831398 .

Aja-Fernández, S., Pieciak, T., Vegas-Sanchez-Ferrero, G., 2015. Spatially variant noise estimation in MRI: A homomorphic approach. Medical Image Analysis 20, $184-197$. 
Aja-Fernández, S., Tristán-Vega, A., Alberola-López, C., 2009. Noise estimation in single- and multiple-coil magnetic resonance data based on statistical models. Magnetic Resonance Imaging 27, 1397-1409.

Aja-Fernández, S., Vegas-Sanchez-Ferrero, G., 2016. Statistical analysis of noise in MRI. Springer Science.

Aja-Fernández, S., Vegas-Sánchez-Ferrero, G., Tristán-Vega, A., 2014. Noise Estimation in Parallel MRI: GRAPPA and SENSE. Magnetic Resonance Imaging 32, 281-290.

Beliakov, G., James, S., 2014. A penalty-based aggregation operator for non-convex intervals. KnowledgeBased Systems 70, 335-344.

Borrelli, P., Palma, G., Comerci, M., Alfano, B., 2014. Unbiased noise estimation and denoising in parallel magnetic resonance imaging, in: IEEE Int. Conf. Acoust. Speech Signal Process. (ICASSP), pp. 1230-1234.

Buades, A., Coll, B., Morel, J.M., 2005. A review of image denoising algorithms, with a new one. Multiscale Modeling \& Simulation 4, 490-530.

Calvo, T., Beliakov, G., 2010. Aggregation functions based on penalties. Fuzzy Sets and Systems 161, $1420-1436$.

Calvo, T., Mesiar, R., Yager, R.R., 2004. Quantitative weights and aggregation. Fuzzy Systems, IEEE Transactions on $12,62-69$.

Cocosco, C.A., Kollokian, V., Kwan, R.K.S., Pike, G.B., Evans, A.C., 1997. Brainweb: Online interface to a $3 \mathrm{D}$ MRI simulated brain database, in: NeuroImage.

Delakis, I., Hammad, O., Kitney, R.I., 2007. Wavelet-based de-noising algorithm for images acquired with parallel magnetic resonance imaging (MRI). Phys. Med. Biol. 52, 3741.

Dietrich, O., Raya, J.G., Reeder, S.B., Ingrisch, M., Reiser, M.F., Schoenberg, S.O., 2008. Influence of multichannel combination, parallel imaging and other reconstruction techniques on MRI noise characteristics. Magnetic Resonance Imaging 26, 754-762.

González-Jaime, L., Kerre, E.E., Nachtegael, M., Bustince, H., 2014. Consensus image method for unknown noise removal. Knowledge-Based Systems 70, 64-77.

González-Jaime, L., Nachtegael, M., Kerre, E.E., Vegas-Sánchez-Ferrero, G., Aja-Fernández, S., 2013. Parametric Image Restoration Using Consensus: An Application to Nonstationary Noise Filtering, in: Sanches, J.M., Micó, L., Cardoso, J.S. (Eds.), Pattern Recognition and Image Analysis. Springer Berlin Heidelberg. volume 7887 of Lecture Notes in Computer Science, pp. 358-365.

Goossens, B., Pizurica, A., Philips, W., 2006. Wavelet Domain Image Denoising for Non-Stationary Noise and Signal-Dependent Noise, in: IEEE International Conference on Image Processing, pp. 1425-1428.

Griswold, M.A., Jakob, P.M., Heidemann, R.M., Nittka, M., Jellus, V., Wang, J.M., Kiefer, B., Haase, A., 2002. Generalized Autocalibrating Partially Parallel Acquisitions (GRAPPA). Magnetic Resonance in Medicine 47, 1202-1210.

Gudbjartsson, H., Patz, S., 1995. The Rician distribution of noisy MRI data. Magnetic Resonance in Medicine 34, 910-914.

Henkelman, R.M., 1985. Measurement of Signal Intensities in the Presence of Noise in MR images. Medical Physics 12, 232+.

Liu, J.P., Lin, S., Chen, H.Y., Xu, Q., 2013. Penalty-based continuous aggregation operators and their application to group decision making. Knowledge-Based Systems 37, 490-501. 
Liu, R.W., Shi, L., Huang, W., Xu, J., Yu, S.C.H., Wang, D., 2014. Generalized total variation-based MRI Rician denoising model with spatially adaptive regularization parameters. Magn. Reson. Imag. 32, $702-720$.

Luisier, F., Blu, T., Wolfe, P.J., 2012. A CURE for noisy magnetic resonance images: Chi-square unbiased risk estimation. Image Processing, IEEE Transactions on 21, 3454-3466.

Maggioni, M., Foi, A., 2012. Nonlocal transform-domain denoising of volumetric data with groupwise adaptive variance estimation, in: IS\&T/SPIE Electronic Imaging, International Society for Optics and Photonics. pp. $82960 \mathrm{O}-82960 \mathrm{O}-8$.

Manjon, J.V., Carbonell-Caballero, J., Lull, J.J., Garcia-Marti, G., Marti-Bonmati, L., Robles, M., 2008. MRI denoising using Non-Local Means. Medical Image Analysis 12, 514-523.

Manjón, J.V., Coupé, P., Buades, A., 2015. MRI noise estimation and denoising using non-local PCA. Medical Image Analysis .

McGibney, G., Smith, M.R., 1993. An unbiased signal-to-noise ratio measure for magnetic-resonance images. Medical Physics 20, 1077-1078.

Pan, X., Zhang, X., Lyu, S., 2012. Blind local noise estimation for medical images reconstructed from rapid acquisition, in: SPIE Medical Imaging, pp. 83143R-83143R.

Pieciak, T., Vegas-Sánchez-Ferrero, G., Aja-Fernández, S., 2016. Variance stabilization of noncentral-chi data: Application to noise estimation in MRI, in: Biomedical Imaging: From Nano to Macro, 2016 IEEE International Symposium on.

Pruessmann, K.P., Weiger, M., Scheidegger, M.B., Boesiger, P., 1999. SENSE: Sensitivity encoding for fast MRI. Magnetic Resonance in Medicine 42, 952-62.

Robson, P.M., Grant, A.K., Madhuranthakam, A.J., Lattanzi, R., Sodickson, D.K., McKenzie, C.A., 2008. Comprehensive quantification of signal-to-noise ratio and g-factor for image-based and k-space-based parallel imaging reconstructions. Magnetic Resonance in Medicine 60, 895-907.

Sijbers, J., den Dekker, A.J., Scheunders, P., Van Dyck, D., 1998. Maximum-likelihood estimation of Rician distribution parameters. Medical Imaging, IEEE Transactions on 17, 357-361.

Tabelow, K., Voss, H.U., Polzehl, J., 2015. Local estimation of the noise level in MRI using structural adaptation. Med. Imag. Anal. 20, 76 - 86 .

Thunberg, P., Zetterberg, P., 2007. Noise distribution in SENSE- and GRAPPA-reconstructed images: a computer simulation study. Magnetic Resonance Imaging 25, 1089-1094.

Tristán-Vega, A., García-Pérez, V., Aja-Fernández, S., Westin, C.F., 2012. Efficient and robust nonlocal means denoising of MR data based on salient features matching. Computer Methods and Programs in Biomedicine 105, 131-144.

Wang, Z., Bovik, A.C., Sheikh, H.R., Simoncelli, E.P., 2004. Image quality assessment: from error visibility to structural similarity. Image Processing, IEEE Transactions on 13, 600-612.

Yager, R.R., 1988. On ordered weighted averaging aggregation operators in multicriteria decision-making. Systems Man and Cybernetics, IEEE Transactions on 18, 183-190. 